\title{
Impact on visual acuity and psychological outcomes of ranibizumab and subsequent treatment for diabetic macular oedema in Japan (MERCURY)
}

\author{
Taiji Sakamoto $^{1}$ - Masahiko Shimura ${ }^{2} \cdot$ Shigehiko Kitano $^{3} \cdot$ Masahito Ohji $^{4} \cdot$ Yuichiro Ogura $^{5}$. \\ Hidetoshi Yamashita ${ }^{6} \cdot$ Makoto Suzaki $^{7} \cdot$ Kimie Mori $^{7} \cdot$ Yohei Ohashi $^{7} \cdot$ Poh Sin Yap ${ }^{8} \cdot$ Takeumi Kaneko $^{7}$. \\ Tatsuro Ishibashi ${ }^{9} \cdot$ for the MERCURY Study Group
}

Received: 5 March 2021 / Revised: 17 June 2021 / Accepted: 29 June 2021 / Published online: 3 September 2021

(c) The Author(s) 2021

\begin{abstract}
Purpose The MERCURY study aimed to evaluate the effects on visual acuity and psychological symptoms, and safety, of ranibizumab and subsequent treatment in patients with diabetic macular oedema (DME) and impaired visual acuity (VA). We report data from the prespecified 12-month interim analysis.

Methods This was a 24-month, phase 4, open-label, single-arm, prospective, observational study conducted at 20 specialised retinal centres in Japan. Participants were 209 patients with DME and impaired VA, not previously treated with either intravitreal or systemic anti-vascular endothelial growth factor (anti-VEGF) agents, who initiated ranibizumab $0.5 \mathrm{mg}$ per investigator discretion. Following ranibizumab administration, patients were treated per routine clinical practice. Other treatments were allowed. The main outcome measure was the mean change in best-corrected VA (BCVA) in logarithmic minimum angle of resolution (logMAR) from baseline to month 12. An exploratory objective was to assess patients' psychological status using the Hospital Anxiety and Depression Scale (HADS).

Results The mean \pm standard deviation BCVA at baseline was $0.43 \pm 0.39 \log$ MAR. The mean number of injections of ranibizumab and anti-VEGF agents from baseline to month 11 was $3.2 \pm 2.0$ and $3.6 \pm 2.4$, respectively. The BCVA change from baseline to 12 months was $-0.08 \pm 0.34 \log$ MAR $(p=0.011)$, showing a significant improvement; the HADS-anxiety score also decreased significantly $(p=0.001)$ and the depression score decreased numerically $(p=0.080)$.

Conclusion MERCURY study data confirm the effectiveness of real-world treatment initiated with ranibizumab in Japanese patients with DME. In addition, treatment was able to positively influence anxiety via VA improvement.
\end{abstract}

\section{Key messages}

- Diabetic macular oedema (DME) is a common cause of vision loss in patients with diabetes, which may be associated with psychological burden.

- We examined the effects of ranibizumab and subsequent treatment in patients with DME and visual impairment on visual acuity and anxiety and depression.

- After 12 months of treatment, mean best-corrected visual acuity significantly improved.

- There was also a significant decrease in anxiety scores, but not in depression scores.

Keywords Anti-VEGF $\cdot$ Diabetic macular oedema $\cdot$ HADS $\cdot$ Ranibizumab $\cdot$ Real-world data $\cdot$ Visual acuity

The full list of the MERCURY study group investigators and advisors are provided in Online Resource 1

Extended author information available on the last page of the article 


\section{Introduction}

Diabetic macular oedema (DME) is the most common cause of vision loss in patients with diabetes [1]. Estimates in 2017 suggested that 451 million people globally are affected by diabetes [2], and up to $15.3 \%$ of them have DME [3]. As the prevalence of diabetes continues to increase [2], the burden of DME is also expected to grow. The current mainstay of treatment for DME is antivascular endothelial growth factor (VEGF) therapy [4-6].

Like other chronic health conditions [7], diabetes is known to be associated with elevated levels of depression and anxiety [8]. In a cross-sectional study of 2,049 patients with diabetes, investigators found evidence for high levels of anxiety and depression (defined as a score $\geq 8$ on the Hospital Anxiety and Depression Scale [HADS]), compared with general population samples [9]. Several subsequent publications have confirmed that HADS scores are higher in patients with diabetic complications than in those without [9-11]. Moreover, studies have indicated that the severity of diabetic retinopathy and/or DME may be associated with poor psychosocial functioning [12] and an elevated level of depression [11]. Poor visual acuity (VA) in the better eye (BE) has also been linked with anxiety and/or depression in other diseases of the eye, including age-related macular degeneration [13, 14] and glaucoma [15]. This is consistent with data which indicate that, in older adults, visual impairment in the BE is associated with a high prevalence of anxiety and depression (measured using HADS and other psychological assessments), compared with normally-sighted peers [16].

It has been demonstrated that it is possible to improve HADS-anxiety (HADS-A) and HADS-depression (HADSD) scores in visually impaired individuals by correcting low vision [17]; however, to date, there have been few reports evaluating the influence of DME treatment on mental status in patients with DME and impaired VA. Moreover, to our knowledge, there have been no studies to assess the psychological impact of DME treatment in patients using a specific psychological screening tool, such as HADS; indeed, other researchers have noted that this is an area of research that would benefit from additional investigation [12].

Ranibizumab, the first approved anti-VEGF agent for DME, is a recombinant humanised monoclonal antibody fragment that binds to all isoforms of VEGF-A [18]. It is administered intravitreally at a dose of $0.5 \mathrm{mg}$ (Europe and Japan [19]) or $0.3 \mathrm{mg}$ (USA) for DME. Phase 3 clinical studies have shown that intravitreal ranibizumab, either as monotherapy or combined with laser treatment, was more effective than laser alone for the treatment of DME over $\geq 1$ year $[20,21]$.
DME is a long-term condition that places a considerable burden on patients and raises several questions regarding optimal treatment. Repeated intravitreal injections are generally required to maintain improved VA in DME patients [22]. However, the standard criteria for defining stability of VA and disease activity, including guidance for the timing of initiation, interruption and retreatment of ocular anti-VEGF agents, are still not well characterised.

The MERCURY study, a prospective, observational study, was designed to evaluate the effectiveness in terms of VA and safety of ranibizumab and subsequent therapy in the real-world clinical setting for patients with DME who had impaired VA. Additionally, the study aimed to assess the psychological impact associated with the expected VA improvement following anti-VEGF treatment in patients with DME.

\section{Methods}

\section{Study design}

This was a 24-month, phase 4, open-label, single-arm, multicentre, prospective, observational study in DME patients with impaired VA in Japan (Online Resource 2; Online Resource 3). The MERCURY study (JapicCTI-173610) has enrolled patients with DME who initiated ranibizumab $0.5 \mathrm{mg}$ in daily clinical practice. This report includes data (including the primary outcome measure) from the prespecified 12-month interim analysis (cut-off date: September 9, 2019).

All participating patients were to receive at least one ranibizumab injection, but this was an observational study to assess safety and effectiveness in routine clinical practice; thus, other treatments were allowed.

The study protocol and informed consent form were reviewed and approved by each centre's institutional review board (Online Resource 4), and all patients or their legal representatives provided written informed consent. The study was conducted in accordance with the principles of the Declaration of Helsinki and the guidelines for Good Clinical Practice, Good Post-marketing Study Practice and Good Pharmacoepidemiology Practices. A detailed study design is provided in Online Resource 3.

\section{Patients}

Patients eligible for inclusion were those with DME who had impaired VA as judged by the investigator; aged $\geq 20$ years; initiating treatment with ranibizumab for the first time and not previously treated with either intravitreal or systemic anti-VEGF agents; and who anticipated being able to visit the study centre for at least 1 year. Exclusion criteria were 
simultaneous participation in other investigational studies; planned treatment with systemic anti-VEGF agents within 1 year from baseline; contraindication or hypersensitivity to ranibizumab or its excipients; or diagnosed or suspected infection, or inflammation, in and/or around the eye.

\section{Treatment}

The only recommendation regarding dose, frequency or duration of treatment was that patients received ranibizumab according to the Japanese package insert [19]. Ranibizumab $(0.5 \mathrm{mg})$ was administered pro re nata (PRN) by intravitreal injection $(0.05 \mathrm{~mL})$ on a monthly basis. From the second injection (month 1), other ocular anti-VEGF agents were allowed at the investigator's discretion. Additional adjunctive treatments (e.g. photocoagulation, intraocular injection of steroid or vitrectomy) were not restricted during the study period.

Patients were able to receive ranibizumab injections in one or both eyes. The first eye receiving a ranibizumab injection was considered the primary treated eye (PTE). If the second eye also received a ranibizumab injection prior to other anti-VEGF agent injections, it was considered the secondary treated eye (STE). If both eyes were treated on the same date, the eye with the earliest diagnosis date was considered the PTE. If both eyes had the same diagnosis date, one eye was chosen as the PTE by the investigator.

\section{Study outcomes and measures}

The primary study objective was to describe the effectiveness of ranibizumab and subsequent treatment in DME patients with impaired VA in clinical settings. This was evaluated by assessing mean change in best-corrected VA (BCVA) in logarithmic minimum angle of resolution (logMAR) from baseline to month 12 .

Secondary objectives were to characterise the effectiveness of ranibizumab and subsequent treatment by evaluating the monthly changes in BCVA in logMAR and central subfield thickness (CSFT) measured by optical coherence tomography over 12 months. A further secondary objective was to characterise safety during the study.

Exploratory objectives were to assess the psychological status of patients using the HADS [23]. The HADS (Japanese translation [24]) was measured at baseline, month 3 and month 12. The HADS is a self-rated questionnaire and consists of seven items for anxiety (HADS-A) and seven items for depression (HADS-D), with a score of 0 to 3 for each. The resulting total score ranges from 0 to 21 for each subscale, with higher scores indicating more severe symptoms; a score of $\geq 8$ indicates subthreshold anxiety or depression. Detailed study outcomes and measures are outlined in Online Resource 3.

\section{Statistical methods}

The planned study size was 200 patients based on prior studies [20, 21]. Further details of sample size calculation, summary statistics and analysis set definitions are outlined in Online Resource 3. Briefly, data were analysed descriptively and summarised together with estimates and corresponding 95\% confidence intervals (CIs) as appropriate. The paired $t$-test was performed to evaluate mean changes and the chi-square test used to compare categorical variables. Pearson's correlation coefficient was used to assess correlations between continuous variables. A $p$ value of $<0.05$ was considered statistically significant.

\section{Results}

\section{Patients}

In total, 209 patients were enrolled; of these, 192 (91.9\%) completed 12 months of observation. The most common reason for discontinuation was a withdrawal of consent $(n=11$ [5.3\%]); no patients discontinued the study due to adverse events (AEs). Full details of patient disposition are shown in Online Resource 5. The safety set comprised all 209 enrolled patients. For effectiveness analyses, the PTE set also contained 209 patients (100\%) and the STE set contained 61 patients $(29.2 \%)$.

Table 1 shows the baseline characteristics of the PTE set. The mean age of patients was 64.4 years and $129(61.7 \%)$ were males. One-third of patients had proliferative diabetic retinopathy $(n=68 ; 32.5 \%)$. Just over half of patients $(n=120 ; 57.4 \%)$ had undergone previous DME treatment prior to this study, including panretinal photocoagulation $(n=69)$, ocular steroid injection $(n=48)$, and laser photocoagulation $(n=36)$. The mean baseline BCVA (logMAR) was 0.43 in the PTE set (equivalent to 63.5 early treatment diabetic retinopathy study [ETDRS] letters). Relevant nonocular disease characteristics such as glycated haemoglobin $(\mathrm{HbA} 1 \mathrm{c})$, blood pressure and medical history are presented in Online Resource 6.

\section{Effectiveness outcomes}

The results for mean change in BCVA from baseline to month 12 for the PTE set are shown in Fig. 1. The BCVA value ( \pm standard deviation [SD]) at baseline was $0.43 \pm 0.39$ $\operatorname{logMAR}$. Significant improvements in BCVA were shown after 3 months and after 12 months, as shown by the change in BCVA from baseline to 3 months $(-0.08 \pm 0.19 \log$ MAR, $p<0.001)$ and from baseline to 12 months $(-0.08 \pm 0.34$ $\log$ MAR, $p=0.011)$. The proportions of patients achieving BCVA improvements of $\leq-0.1, \leq-0.2$ and $\leq-0.3$ 
Table 1 Baseline patient demographics, and disease and ocular characteristics (PTE set)

\begin{tabular}{ll}
\hline Variable & PTE, $N=209$ \\
\hline Age (years), mean \pm SD & $64.4 \pm 12.8$ \\
Sex, $n(\%)$ & \\
Male & $129(61.7)$ \\
Female & $80(38.3)$ \\
Diabetes type, $n(\%)$ & \\
Type I & $4(1.9)$ \\
Type II & $205(98.1)$ \\
Time since first diagnosis of diabetes (years), & $13.0 \pm 11.1$ \\
mean \pm SD & \\
BCVA (logMAR), mean \pm SD & $0.43 \pm 0.39$ \\
CSFT ( $\mu$ m), $n$ & 203 \\
Mean \pm SD & $459.0 \pm 138.7$ \\
Time from DME diagnosis (years), $n$ & 170 \\
Mean \pm SD & $0.79 \pm 1.74$ \\
Type of DME, $n$ & 204 \\
Unilateral, $n(\%)$ & $82(39.2)$ \\
Bilateral, $n(\%)$ & $122(58.4)$ \\
Lens status (phakic), $n(\%)$ & $125(59.8)$ \\
Classification of diabetic retinopathy, $n(\%)$ & \\
Mild NPDR & $20(9.6)$ \\
Moderate NPDR & $56(26.8)$ \\
Severe NPDR & $59(28.2)$ \\
PDR & $68(32.5)$ \\
Prior DME treatment, $n(\%)$ & $120(57.4)$ \\
Any DME treatment & $36(17.2)$ \\
Grid/focal laser photocoagulation & $48(23.0)$ \\
Intravitreal/subtenon steroid injection & $5(2.4)$ \\
Vitrectomy & $69(33.0)$ \\
Panretinal photocoagulation & $10(4.8)$ \\
Other & \\
\hline
\end{tabular}

BCVA, best-corrected visual acuity; CSFT, central subfield thickness; DME, diabetic macular oedema; logMAR, logarithm of the minimum angle of resolution; NPDR, non-proliferative diabetic retinopathy; PDR, proliferative diabetic retinopathy; PTE, primary treated eye; $\mathrm{SD}$, standard deviation

$\log$ MAR units, and the proportion experiencing BCVA deterioration of $\geq 0.3 \operatorname{logMAR}$ units from baseline to month 12 are shown in Online Resource 7.

The mean change \pm standard error in CSFT from baseline to month 12 for the PTE set is shown in Online Resource 8. Significant differences from baseline were recorded after 3 months $(-81.5 \mu \mathrm{m} \pm 149.0 ; p<0.001)$ and 12 months $(-100.3 \mu \mathrm{m} \pm 145.1 ; p<0.001)$.

\section{Treatment exposure}

The number and frequency of anti-VEGF injections in the PTE and STE sets are shown in Online Resource 9. Overall, the mean \pm SD number of injections of ranibizumab and anti-VEGF agents (including ranibizumab) from baseline to month 11 was $3.2 \pm 2.0$ and $3.6 \pm 2.4$, respectively, in the PTE set. There were 91 injections of anti-VEGF agents other than ranibizumab administered in the PTE set; almost all ( $n=90)$ were aflibercept, and one was bevacizumab. The number of eyes in the PTE set with adjunctive therapy during the same period was $88(42.1 \%)$.

\section{Visual acuity outcomes and treatment frequency or systemic factor}

In the PTE group, patients who received three anti-VEGF injections during the first 2 months demonstrated better BCVA improvement compared with those who received one or two injections during the same period (Fig. 2). This improvement was irrespective of the total number of injections administered during the study.

\section{Exploratory outcomes}

The mean changes in HADS score from baseline to months 3 and 12, and the averaged values over time, are described in Fig. 3. The mean \pm SD baseline HADS-A and HADSD scores were $4.26 \pm 3.79$ and $4.67 \pm 4.22$, respectively. At month 3, the HADS-A score decreased significantly by $-0.76 \pm 2.81(p<0.001)$, with the decrease continuing at month $12(-0.96 \pm 3.13 ; p=0.001)$. At months 3 and 12 , the HADS-D score decreased by $-0.46 \pm 3.16(p=0.053)$ and $-0.54 \pm 3.26(p=0.080)$, respectively.

Overall, 37/206 (18.0\%) and 41/206 (19.9\%) cases had subthreshold anxiety and depression (scores of $\geq 8$ ), respectively. There was a downward trend in anxiety over time, with subthreshold anxiety present in 24/182 (13.2\%) and $14 / 112(12.5 \%)$ patients at months 3 and 12, respectively. Subthreshold depression did not notably alter following treatment; 32/182 (17.6\%) and 22/112 (19.6\%) patients had subthreshold depression at months 3 and 12, respectively.

To investigate the impact of DME treatment on HADSA scores, we evaluated the relationship between HADS and BCVA of the BE (BE BCVA) and between HADS and the number of anti-VEGF injections. At baseline, of the 209 eyes in the PTE set, $44(21.1 \%)$ were classed as the BE, $131(62.7 \%)$ as the worse eye and $25(12.0 \%)$ as having equivalent vision with the other eye; data for nine eyes $(4.3 \%)$ were missing. In the STE set, the numbers were $35(57.4 \%), 14(23.0 \%)$ and $12(19.7 \%)$, respectively; no data were missing for this group. Mean BCVA in the BE at baseline was $0.21 \pm 0.34 \log$ MAR, and in the worse eye was $0.52 \pm 0.45 \log$ MAR (Online Resource 10). After 12 months of treatment to both eyes, BE BCVA improved significantly from baseline $(-0.06 \pm 0.26 \mathrm{log}-$ MAR; $p=0.013)$. Changes in the HADS-A score were 
Fig. 1 Mean \pm SE change in BCVA (logMAR) over time (PTE set). $p$ values were calculated using a 1-sample paired $t$-test versus baseline values. ${ }^{\text {a }}$ The number of eyes at each month corresponds to the number of patients who attended the study visit and were measured for visual acuity at the corresponding month. BCVA, best-corrected visual acuity; $\log$ MAR, logarithm of the minimum angle of resolution; PTE, primary treated eye; SE, standard error

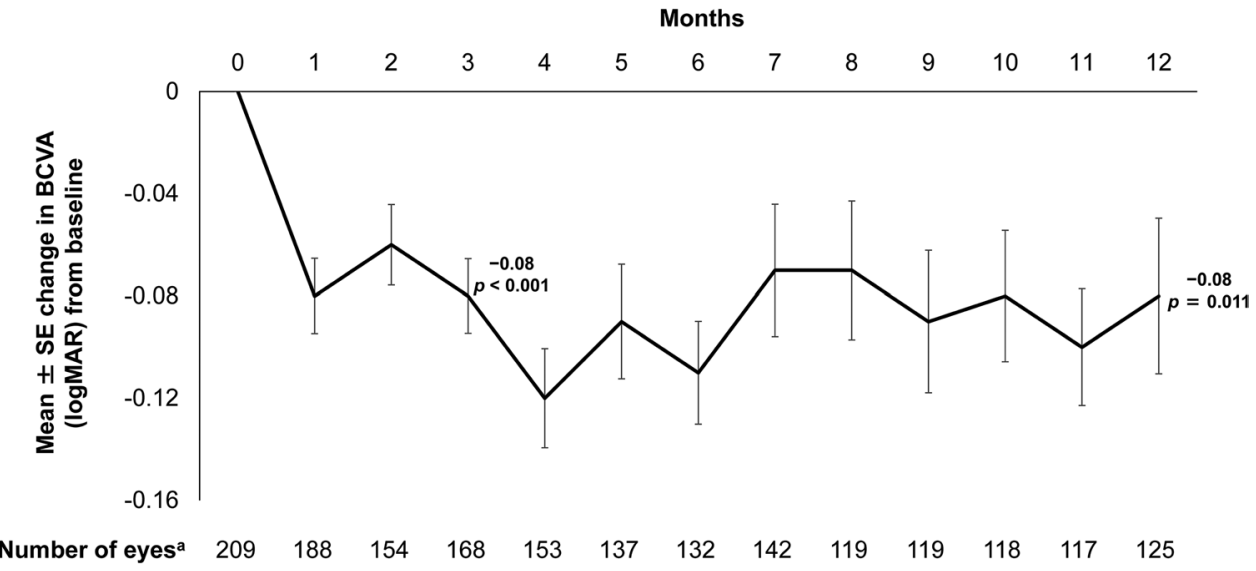

evaluated according to BE BCVA changes (improved or maintained/deteriorated). HADS-A scores significantly decreased from baseline to month 12 in the improved group $(-1.35 \pm 3.43 ; p=0.008)$ but not in the maintained/ deteriorated group $(-0.53 \pm 2.85 ; p=0.191)$ (Table 2$)$. An additional subgroup analysis was conducted for patients who received DME treatment only for the PTE during the first year $(n=62)$. The results demonstrated a similar trend to those of the overall PTE set, with a significant decrease in the HADS-A scores in the improved group $(-1.56 \pm 3.36 ; p=0.023)$ but not in the maintained/ worsened group $(-0.97 \pm 3.08 ; p=0.096)$. There was no correlation between the number of anti-VEGF injections for both eyes from baseline to month 11 and changes in the HADS-A score from baseline to month $12(r=0.027$; $p=0.778$ ) (Online Resource 11).

\section{Safety}

The safety data are summarised in Online Resource 12 . Overall, 19 patients $(9.1 \%)$ in the safety set reported ocular serious AEs (SAEs), of which one event of vitreous haemorrhage was suspected to be related to ranibizumab.



\begin{tabular}{|c|c|c|c|c|c|c|}
\hline \multirow{2}{*}{$\begin{array}{l}\text { Number of anti-VEGF injections during } \\
\text { the first } 2 \text { months } \\
\text { Number of anti-VEGF injections from } \\
\text { baseline to month } 11\end{array}$} & \multicolumn{3}{|c|}{$\begin{array}{c}1-2 \\
(N=157)\end{array}$} & \multicolumn{3}{|c|}{$\begin{array}{c}3 \\
(N=52)\end{array}$} \\
\hline & $\begin{array}{c}1-2 \\
(n=82)\end{array}$ & $\begin{array}{c}3-4 \\
(n=43)\end{array}$ & $\begin{array}{c}5+ \\
(n=32)\end{array}$ & $\begin{array}{c}1-2 \\
(n=0)\end{array}$ & $\begin{array}{c}3-4 \\
(n=18)\end{array}$ & $\begin{array}{c}5+ \\
(n=34)\end{array}$ \\
\hline \multicolumn{7}{|l|}{ BCVA (logMAR), mean $\pm S D$} \\
\hline Baseline & $0.47 \pm 0.45$ & $0.41 \pm 0.31$ & $0.38 \pm 0.33$ & NA & $0.37 \pm 0.39$ & $0.45 \pm 0.39$ \\
\hline Change from baseline to month 12 & $-0.08 \pm 0.45$ & $0.03 \pm 0.31$ & $-0.10 \pm 0.24$ & NA & $-0.18 \pm 0.24$ & $-0.12 \pm 0.26$ \\
\hline$p$ value (versus baseline) & 0.267 & 0.630 & 0.051 & NA & 0.054 & 0.023 \\
\hline
\end{tabular}

Fig. 2 Mean change in BCVA (logMAR) from baseline to month 12 according to the number of anti-VEGF injections administered during the first 2 months (PTE set). Mean BCVA (logMAR) values at baseline were 0.43 and 0.42 in patients who received 1-2 injections from baseline to month 2 and in patients who received 3 injections from baseline to month 2 , respectively. In patients who received 1-2 injections from baseline to month 2 , the mean number of total injec- tions was 3.0. In patients who received 3 injections from baseline to month 2, the mean number of total injections was 5.7. $p$ values were calculated using the paired $t$-test versus baseline values. BCVA, bestcorrected visual acuity; logMAR, logarithm of the minimum angle of resolution; NA, not applicable; PTE, primary treated eye; SD, standard deviation; SE, standard error; VEGF, vascular endothelial growth factor 
Fig. 3 Mean change in HADS scores from baseline (safety set). ${ }^{a} p$ values were calculated using the paired $t$-test versus baseline values. ${ }^{\mathrm{b}} p$ values were calculated using the chi-square test versus baseline values. HADS, Hospital Anxiety and Depression Scale; HADSA, HADS anxiety subscale; HADS-D, HADS depression subscale; IQR, interquartile range; $\mathrm{SD}$, standard deviation; SE, standard error

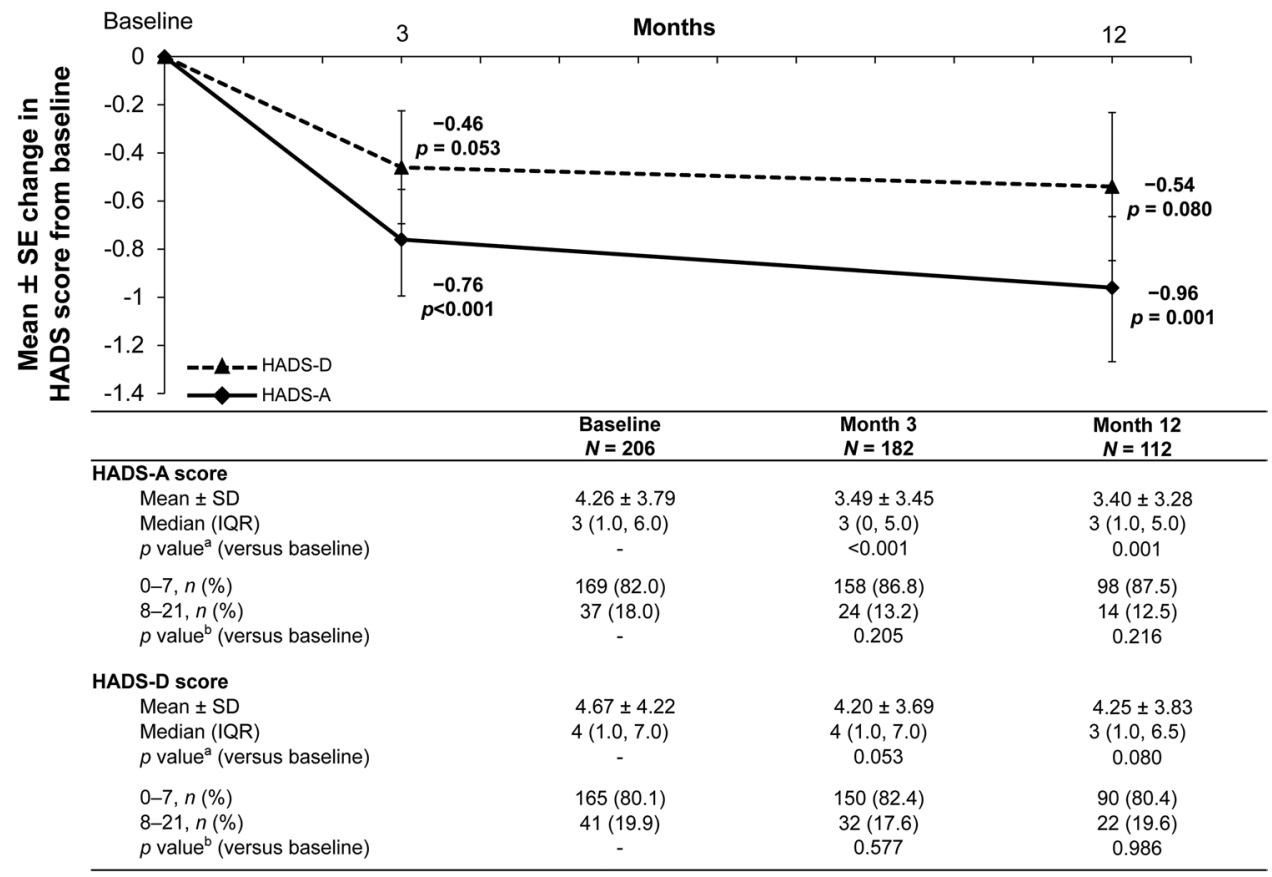

In contrast, 29 patients (13.9\%) reported non-ocular SAEs, of which one event of cerebral infarction was suspected to be related to ranibizumab. Three deaths were reported during the study and were caused by cardiac failure, suicidal behaviour and myocardial infarction; none was

Table 2 HADS change from baseline to month 12 according to BE BCVA (logMAR) change (safety set)

\begin{tabular}{|c|c|c|c|}
\hline HADS score & Improved group $^{\mathrm{a}}, n=54$ & $\begin{array}{l}\text { Maintained/deteriorated group }{ }^{\mathrm{b}} \text {, } \\
n=53\end{array}$ & $\begin{array}{l}p \text { value } \\
\text { (between } \\
\text { group) }\end{array}$ \\
\hline \multicolumn{4}{|l|}{ HADS-A } \\
\hline Baseline, $n$ & 54 & 53 & \\
\hline Mean \pm SD & $4.41 \pm 3.86$ & $4.36 \pm 3.80$ & \\
\hline Change from baseline to month $12, n^{c}$ & 49 & 51 & \\
\hline Mean \pm SD & $-1.35 \pm 3.43$ & $-0.53 \pm 2.85$ & 0.197 \\
\hline $95 \% \mathrm{CI}$ & $(-2.33,-0.36)$ & $(-1.33,0.27)$ & \\
\hline$p$ value (vs baseline) & 0.008 & 0.191 & \\
\hline \multicolumn{4}{|l|}{ HADS-D } \\
\hline Baseline, $n$ & 54 & 53 & \\
\hline Mean \pm SD & $4.56 \pm 4.05$ & $4.72 \pm 4.06$ & \\
\hline Change from baseline to month $12, n^{c}$ & 49 & 51 & \\
\hline Mean \pm SD & $-0.90 \pm 3.37$ & $-0.45 \pm 3.36$ & 0.508 \\
\hline $95 \% \mathrm{CI}$ & $(-1.87,0.07)$ & $(-1.40,0.49)$ & \\
\hline$p$ value (vs baseline) & 0.068 & 0.343 & \\
\hline
\end{tabular}

${ }^{\mathrm{a}}$ Improved = BE BCVA (logMAR) change from baseline to month 12 was $<0$

${ }^{\mathrm{b}}$ Maintained/deteriorated $=$ BE BCVA (logMAR) change from baseline to month 12 was $\geq 0$

${ }^{\mathrm{c}}$ Patients with evaluable data at baseline and month 12

Nominal $p$ values (vs baseline) were calculated using the paired $t$-test. Nominal $p$ values between the groups were calculated using a 2 -sample $t$-test

The eye with better BCVA (higher decimal or lower logMAR) compared with the opposite eye was considered as the 'better eye'

BE BCVA, better eye best-corrected visual acuity; CI, confidence interval; HADS, Hospital Anxiety and Depression Scale; HADS-A, HADS anxiety subscale; HADS-D, HADS depression subscale; logMAR, logarithm of the minimum angle of resolution; SD, standard deviation 
suspected to be related to ranibizumab. Details of ocular and non-ocular SAEs are provided in Online Resource 13.

\section{Discussion}

These results from the MERCURY study, a real-world, observational analysis of Japanese patients with DME and impaired VA, show that 12 months after initiation of ranibizumab treatment, mean BCVA (logMAR) values were significantly improved $(p=0.011)$, as was CSFT $(p<0.001)$. Over the same duration, the HADS-A score decreased significantly $(p=0.001)$ and the HADS-D score decreased numerically $(p=0.080)$. To the best of our knowledge, this is the first study to assess the psychological effects of anti-VEGF treatments for DME patients using the HADS, a specific psychological measure.

The mean BCVA gain at month 12 in MERCURY (mean change $-0.08 \log$ MAR, equivalent to +4 ETDRS letters) is comparable with results from other real-world studies (Online Resource 14). In two retrospective studies of 12 months' duration, the mean VA gains were +6.6 letters [25] and +4.7 [26] letters with a mean of 7.2 and 3.1 injections, respectively. In the prospective real-world OCEAN [27], BOREAL-DME [28] and LUMINOUS [29] studies, the mean VA gains during 12 months were +4 letters, +7.4 letters and +3.5 letters, achieved with a mean of 4.4, 5.1 and 4.5 injections, respectively. Moreover, other studies with longer duration have reported improvements of +6.6 letters with a mean of 7.7 injections over 4 years [30] and +2 letters with a mean of 3.8 injections over 2 years [31].

Conversely, we can see that the number of injections and the BCVA gains observed in MERCURY and in other real-world studies was slightly lower compared with the results reported from randomised, controlled trials [20, 21]. This is consistent with a previous report which suggested that use of ranibizumab in routine clinical practice resulted in less frequent injections and reduced effectiveness compared with the drug registration trials [32]. It is difficult to achieve adequate treatment with anti-VEGF agents in real-world settings, and one reason for this appears to be the cost of therapy. Based on the results of a questionnaire evaluating anti-VEGF therapy, $85.8 \%$ of physicians responded that financial burden was an important factor which influenced the rate of continuation of injections [33]. Additionally, the fact that patients in MERCURY had relatively high baseline BCVA (Online Resource 14) and well-managed diabetic status (i.e. HbA1c; Online Resource 6) may have resulted in less frequent injections and comparative undertreatment in this study in relation to other trials. Interestingly, our study showed that patients who had three injections during the first 2 months had improved BCVA compared with those who did not. This improvement was observed irrespective of the overall number of injections from baseline to month 11. This regimen, of three injections during the first 2 months, has previously been shown to provide benefit to patients [29] and might be an alternative (and less costly) solution for DME patients in real-world settings.

Previous studies have suggested that the prevalences of anxiety and depression are higher in older patients with poor VA [16, 34], in patients with diabetes [10] and in those with diabetic ocular complications $[11,12,35]$. Of note, Rees et al. [11] reported that $24.3 \%$ and $16.3 \%$ of patients with DME, respectively, had HADS-A and HADS-D scores of $\geq 8$. This is comparable to our results showing that $18.0 \%$ and $19.9 \%$, respectively, had HADS-A and HADS-D scores of $\geq 8$. However, our results also showed that the HADS-A score decreased significantly $(p=0.001)$ and the HADSD score decreased numerically $(p=0.080)$ following DME treatment. Furthermore, a downward trend in the number of patients with subthreshold anxiety was also observed during the study. Visual impairment in the BE is known to increase the prevalence of subthreshold anxiety and correlate with increased HADS-A scores $[14,16]$.

In our stratified analysis of BE BCVA, the HADS-A score significantly decreased in the improved group, but not in the maintained/deteriorated group. This suggests that improvement in BE BCVA following DME treatment was able to lessen the anxiety symptoms felt by the patients in our study. Additionally, the results from the subgroup analysis of patients who received DME treatment only for PTE was consistent with the data from the overall patient population. That the same trend was observed in the subgroup supports the premise that improvement in BE BCVA following DME treatment is associated with a lessening of anxiety symptoms. However, there was no significant correlation between the HADS-A score and the number of anti-VEGF injections. We consider that there is a low possibility that changes in systemic factors of patients affected the HADS scores during our study, based on a previous report that the values of systemic factors, such as blood glucose, blood pressure and lipids, were unrelated to the HADS-A score in patients with diabetic retinopathy [11]. Thus, DME treatment initiated with ranibizumab may provide additional benefit to patients with DME in the real-world setting by diminishing psychological symptoms via the improvement of VA.

Notably, the reason for the differing results according to HADS-A and HADS-D in the study was unclear. It has been reported that deteriorated BE BCVA worsens the scores of both HADS subscales [14,34], but there is a lack of detailed clinical data, and further investigations are needed to clarify this point. In our study, there was no change in HADS-D scores in patients with DME after 1 year of treatment, and this result is in line with the previous study by Rees et al. 
[11], in which no correlation between DME and HADS-D was reported.

\section{Limitations}

The potential limitations of the study are primarily due to its observational study design, but it must be remembered that this type of study is best suited to obtain real-life data. Although the use of the Landolt $\mathrm{C}$ chart was recommended to assess BCVA, the investigators were allowed to measure vision per their usual practice; thus, there may have been variability in the quality of visual acuity measurements. The lack of a comparator arm means that the effectiveness and safety of ranibizumab treatment cannot be directly compared with other studies of interventional therapy. Moreover, as with all measures relying on subjective patient responses, the use of psychological questionnaires such as HADS presents some difficulties in proving statistically significant changes in mental symptoms by treatment or between groups. Finally, the variable visit schedule of each patient means that there was a much lower rate of data available for evaluation at month 12 (as described in the footnotes of each figure) than the discontinuation rate would suggest. Most patients visited the clinic and underwent evaluation every few months. However, since the fluctuation of BCVA change at each month was small, we consider the results from the patients assessed at month 12 to be indicative of the overall population.

\section{Conclusions}

Data from the open-label, observational MERCURY study confirm the effectiveness and safety of DME treatment initiated with ranibizumab in Japanese patients. In addition, treatment was able to provide potential positive effects on anxiety via VA improvement.

Supplementary Information The online version contains supplementary material available at https://doi.org/10.1007/s00417-021-05308-8.

Acknowledgements The authors thank all investigators who participated at each institute in this study. The authors also thank Michihiro Shono, a former employee of Novartis Pharma K.K. (Tokyo, Japan), Sadhu Sanchayita (Novartis Healthcare Pvt. Ltd., Hyderabad, India) and Rajendra Prasad Sarkar (Novartis Corporation Sdn. Bhd., Selangor, Malaysia), for preparing the study protocol and implementing the statistical analyses. We thank Sally-Anne Mitchell, PhD, of Edanz Pharma, for providing medical writing support, which was funded by Novartis Pharma K.K. through EMC K.K. in accordance with Good Publication Practice (GPP3) guidelines (http://www.ismpp.org/gpp3).

The full list of the MERCURY study group investigators and advisors is provided in Online Resource 1.

Author contribution All authors attest that they meet the current ICMJE criteria for authorship. Conceptualisation: Taiji Sakamoto,
Masahiko Shimura, Shigehiko Kitano, Masahito Ohji, Yuichiro Ogura, Hidetoshi Yamashita, Makoto Suzaki, Kimie Mori, Yohei Ohashi, Takeumi Kaneko and Tatsuro Ishibashi; data curation: Makoto Suzaki, Kimie Mori and Poh Sin Yap; formal analysis: Poh Sin Yap; funding acquisition: Makoto Suzaki, Kimie Mori, Yohei Ohashi and Takeumi Kaneko; investigation: Taiji Sakamoto, Masahiko Shimura, Shigehiko Kitano, Masahito Ohji, Yuichiro Ogura, Hidetoshi Yamashita, Makoto Suzaki, Kimie Mori, Yohei Ohashi, Takeumi Kaneko and Tatsuro Ishibashi; methodology: Taiji Sakamoto, Masahiko Shimura, Shigehiko Kitano, Masahito Ohji, Yuichiro Ogura, Hidetoshi Yamashita, Makoto Suzaki, Kimie Mori, Yohei Ohashi, Takeumi Kaneko, Poh Sin Yap and Tatsuro Ishibashi; project administration: Makoto Suzaki, Kimie Mori, Yohei Ohashi and Takeumi Kaneko; resources: Taiji Sakamoto, Masahiko Shimura, Shigehiko Kitano, Masahito Ohji, Yuichiro Ogura, Hidetoshi Yamashita and Tatsuro Ishibashi; supervision: Taiji Sakamoto, Masahiko Shimura, Shigehiko Kitano, Masahito Ohji, Yuichiro Ogura, Hidetoshi Yamashita and Tatsuro Ishibashi; visualisation: Taiji Sakamoto, Makoto Suzaki, Kimie Mori, Yohei Ohashi and Takeumi Kaneko; writing_-original draft: Taiji Sakamoto, Makoto Suzaki and Kimie Mori; writing — review \& editing: Taiji Sakamoto, Masahiko Shimura, Shigehiko Kitano, Masahito Ohji, Yuichiro Ogura, Hidetoshi Yamashita, Makoto Suzaki, Kimie Mori, Yohei Ohashi, Takeumi Kaneko, Poh Sin Yap and Tatsuro Ishibashi.

Funding This study was funded and sponsored by Novartis Pharma K.K., Tokyo, Japan, which had a role in study design, data collection, data analysis, interpretation of data and development of the manuscript.

Data Availability The data relevant to the study findings are included in the article or uploaded as supplementary information. No additional data are available.

Code availability Not applicable.

\section{Declarations}

Ethics approval The study protocol and informed consent form were reviewed and approved by each centre's institutional review board. This study was conducted in accordance with the principles of the Declaration of Helsinki and the guidelines for Good Clinical Practice, Good Post-marketing Study Practice and Good Pharmacoepidemiology Practices.

Consent to participate All patients or their legal representatives provided written informed consent.

Consent for publication Not applicable.

Conflict of interest Taiji Sakamoto reports personal fees from Novartis Pharma K.K. (Tokyo, Japan) for this study; has worked as a consultant for Bayer (Osaka, Japan) and received personal fees; has received grant support from Chugai (Tokyo, Japan), Senju (Osaka, Japan), Novartis Pharma K.K. (Tokyo, Japan) and Parexel (Tokyo, Japan); and has received personal fees (lecture fees) from Santen (Osaka, Japan), Bayer (Osaka, Japan), Novartis Pharma K.K. (Tokyo, Japan), Alcon (Tokyo, Japan), Otsuka (Tokyo, Japan), Senju (Osaka, Japan), Kowa (Aichi, Japan), Wakamoto (Tokyo, Japan), MSD (Tokyo, Japan), Bausch \& Lomb (Tokyo, Japan), Chuo Sangiyo (Hyogo, Japan), Japan Ophthalmic Instruments Association (Tokyo, Japan), Parexel (Tokyo, Japan), Nidek (Aichi, Japan), R E Medical (Osaka, Japan), KY CenterVue (Tokyo, Japan) and Chugai (Tokyo, Japan) outside the submitted work. Masahiko Shimura has received grant support from Novartis Pharma K.K. (Tokyo, Japan) and Bayer (Osaka, Japan) outside the submitted work. Shigehiko Kitano has received personal fees from Novartis 
Pharma K.K. (Tokyo, Japan) for this study; grant support from Novartis Pharma K.K. (Tokyo, Japan), Bayer (Osaka, Japan), Senju (Osaka, Japan), Santen (Osaka, Japan), Alcon (Tokyo, Japan) and Otsuka (Tokyo, Japan); and personal fees (lecture fees) from Novartis Pharma K.K. (Tokyo, Japan), Kowa (Aichi, Japan), MSD (Tokyo, Japan), Bayer (Osaka, Japan) and Novo Nordisk (Tokyo, Japan) outside the submitted work. Masahito Ohji has received personal fees from Novartis Pharma K.K. (Tokyo, Japan) for this study; personal fees for work as a consultant for Allergan (Tokyo, Japan), Bayer (Osaka, Japan), HOYA (Tokyo, Japan), Novartis Pharma K.K. (Tokyo, Japan), Chengdu Kanghong Biotechnology (Chengdu, China) and Alcon (Tokyo, Japan); grant support from Santen (Osaka, Japan), Novartis Pharma K.K. (Tokyo, Japan), Alcon (Tokyo, Japan), Otsuka (Tokyo, Japan), Senju (Osaka, Japan), Pfizer (Tokyo, Japan), HOYA (Tokyo, Japan) and AMO (Tokyo, Japan); and personal fees (lecture fees) from Santen (Osaka, Japan), Novartis Pharma K.K. (Tokyo, Japan), Bayer (Tokyo, Japan), Alcon (Tokyo, Japan), Otsuka (Tokyo, Japan), Kowa (Aichi, Japan) and Senju (Osaka, Japan) outside the submitted work. Yuichiro Ogura has received personal fees from Novartis Pharma K.K. (Tokyo, Japan) for this study; personal fees for work as a consultant for Bayer (Osaka, Japan), Novartis Pharma K.K. (Tokyo, Japan), HOYA (Tokyo, Japan), Kowa (Aichi, Japan), Astellas (Tokyo, Japan), Janssen (Tokyo, Japan), Alcon (Tokyo, Japan), Graybug (CA, USA), Kyoto Drug Discovery \& Development (Kyoto, Japan), Allergan (Tokyo, Japan) and Chugai (Tokyo, Japan); grant support from Boehringer Ingelheim (Tokyo, Japan) and Novartis Pharma K.K. (Tokyo, Japan); and personal fees (lecture fees) from Bayer (Osaka, Japan), Novartis Pharma K.K. (Tokyo, Japan), Santen (Osaka, Japan), Senju (Osaka, Japan), Sanwa Kagaku (Aichi, Japan), Wakamoto (Tokyo, Japan), Otsuka (Tokyo, Japan), Topcon (Tokyo, Japan) and Nikon (Tokyo, Japan) outside the submitted work. Hidetoshi Yamashita has received personal fees from Novartis Pharma K.K. (Tokyo, Japan) for this study; grant support from Novartis Pharma K.K. (Tokyo, Japan), Alcon (Tokyo, Japan), Santen (Osaka, Japan), Senju (Osaka, Japan), AMO (Tokyo, Japan), Trust Medical (Miyagi, Japan) and Atsuzawa Prosthesis (Tokyo, Japan); and personal fees (lecture fees) from Novartis Pharma K.K. (Tokyo, Japan), Santen (Osaka, Japan), Senju (Osaka, Japan), Bausch \& Lomb (Tokyo, Japan) and Bayer (Osaka, Japan) outside the submitted work. Makoto Suzaki, Kimie Mori, Yohei Ohashi and Takeumi Kaneko are employees of Novartis Pharma K.K. (Tokyo, Japan). Poh Sin Yap is an employee of Novartis Corporation Sdn. Bhd. (Selangor, Malaysia). Tatsuro Ishibashi has received personal fees from Novartis Pharma K.K. (Tokyo, Japan) for this study and lecture fees from Santen (Osaka, Japan), Bayer (Osaka, Japan), Otsuka (Tokyo, Japan), Bausch \& Lomb (Tokyo, Japan), Rohto (Osaka, Japan), Senju (Osaka, Japan) and Novartis Pharma K.K. (Tokyo, Japan) outside the submitted work.

Open Access This article is licensed under a Creative Commons Attribution 4.0 International License, which permits use, sharing, adaptation, distribution and reproduction in any medium or format, as long as you give appropriate credit to the original author(s) and the source, provide a link to the Creative Commons licence, and indicate if changes were made. The images or other third party material in this article are included in the article's Creative Commons licence, unless indicated otherwise in a credit line to the material. If material is not included in the article's Creative Commons licence and your intended use is not permitted by statutory regulation or exceeds the permitted use, you will need to obtain permission directly from the copyright holder. To view a copy of this licence, visit http://creativecommons.org/licenses/by/4.0/.

\section{References}

1. Bandello F, Battaglia Parodi M, Lanzetta P, Loewenstein A, Massin P, Menchini F, Veritti D (2017) Diabetic macular edema. Dev Ophthalmol 58:102-138. https://doi.org/10.1159/000455277

2. Cho NH, Shaw JE, Karuranga S, Huang Y, da Rocha Fernandes JD, Ohlrogge AW, Malanda B (2018) IDF Diabetes Atlas: global estimates of diabetes prevalence for 2017 and projections for 2045. Diabetes Res Clin Pract 138:271-281. https://doi.org/10. 1016/j.diabres.2018.02.023

3. Thomas RL, Halim S, Gurudas S, Sivaprasad S, Owens DR (2019) IDF Diabetes Atlas: a review of studies utilising retinal photography on the global prevalence of diabetes related retinopathy between 2015 and 2018. Diabetes Res Clin Pract 157:107840. https://doi.org/10.1016/j.diabres.2019.107840

4. Browning DJ, Stewart MW, Lee C (2018) Diabetic macular edema: evidence-based management. Indian J Ophthalmol 66:1736-1750. https://doi.org/10.4103/ijo.IJO_1240_18

5. Schmidt-Erfurth U, Garcia-Arumi J, Bandello F, Berg K, Chakravarthy U, Gerendas BS, Jonas J, Larsen M, Tadayoni R, Loewenstein A (2017) Guidelines for the management of diabetic macular edema by the European Society of Retina Specialists (EURETINA). Ophthalmologica 237:185-222. https:// doi.org/10.1159/000458539

6. Terasaki H, Ogura Y, Kitano S, Sakamoto T, Murata T, Hirakata A, Ishibashi T (2018) Management of diabetic macular edema in Japan: a review and expert opinion. Jpn J Ophthalmol 62:123. https://doi.org/10.1007/s10384-017-0537-6

7. National Institute of Mental Health, US National Institutes of Health (2015) Chronic illness and mental health: recognizing and treating depression. https://www.nimh.nih.gov/health/publi cations/chronic-illness-mental-health/

8. Ducat L, Philipson LH, Anderson BJ (2014) The mental health comorbidities of diabetes. JAMA 312:691-692. https://doi.org/ 10.1001/jama.2014.8040

9. Collins MM, Corcoran P, Perry IJ (2009) Anxiety and depression symptoms in patients with diabetes. Diabet Med 26:153161. https://doi.org/10.1111/j.1464-5491.2008.02648.x

10. Bouhassira D, Letanoux M, Hartemann A (2013) Chronic pain with neuropathic characteristics in diabetic patients: a French cross-sectional study. PLoS One 8:e74195. https://doi.org/10. 1371/journal.pone.0074195

11. Rees G, Xie J, Fenwick EK, Sturrock BA, Finger R, Rogers SL, Lim L, Lamoureux EL (2016) Association between diabetes-related eye complications and symptoms of anxiety and depression. JAMA Ophthalmol 134:1007-1014. https://doi.org/ 10.1001/jamaophthalmol.2016.2213

12. Khoo K, Man REK, Rees G, Gupta P, Lamoureux EL, Fenwick EK (2019) The relationship between diabetic retinopathy and psychosocial functioning: a systematic review. Qual Life Res 28:2017-2039. https://doi.org/10.1007/s11136-019-02165-1

13. Augustin A, Sahel JA, Bandello F, Dardennes R, Maurel F, Negrini C, Hieke K, Berdeaux G, the MICMAC Study Group (2007) Anxiety and depression prevalence rates in age-related macular degeneration. Invest Ophthalmol Vis Sci 48:14981503. https://doi.org/10.1167/iovs.06-0761

14. Inan S, Cetinkaya E, Duman R, Dogan I, Übeyt Inan U (2019) Quality of life among patients with age-related severe macular degeneration assessed using the NEI-VFQ, HADS-A, HADSD and SF-36 tests. A cross-sectional study. Sao Paulo Med J 137:25-32. https://doi.org/10.1590/1516-3180.2018.01950 71218

15. Zhang D, Fan Z, Gao X, Huang W, Yang Q, Li Z, Lin M, Xiao H, Ge J (2018) Illness uncertainty, anxiety and depression in Chinese 
patients with glaucoma or cataract. Sci Rep 8:11671. https://doi. org/10.1038/s41598-018-29489-1

16. van der Aa HPA, Comijs HC, Penninx BWJH, van Rens GHMB, van Nispen RMA (2015) Major depressive and anxiety disorders in visually impaired older adults. Invest Ophthalmol Vis Sci 56:849-854. https://doi.org/10.1167/iovs.14-15848

17. Rishi P, Rishi E, Maitray A, Agarwal A, Nair S, Gopalakrishnan S (2017) Hospital anxiety and depression scale assessment of 100 patients before and after using low vision care: a prospective study in a tertiary eye-care setting. Indian J Ophthalmol 65:1203-1208. https://doi.org/10.4103/ijo.IJO_436_17

18. LUCENTIS $^{\circledR}$ (ranibizumab) solution/kit for intravitreal injection $10 \mathrm{mg} / \mathrm{mL}$ [Package insert] [In Japanese]. Novartis Pharma K.K., Tokyo, Japan; 2019. https://pins.japic.or.jp/pdf/newPINS/00062 995.pdf. Accessed 20 October 2020.

19. Ishibashi T, Li X, Koh A, Lai TYY, Lee FL, Lee WK, Ma Z, Ohji M, Tan N, Cha SB, Shamsazar J, Yau CL, REVEAL Study Group (2015) The REVEAL study: Ranibizumab monotherapy or combined with laser versus laser monotherapy in Asian patients with diabetic macular edema. Ophthalmology 122:1402-1415. https://doi.org/10.1016/j.ophtha.2015.02.006

20. Dervenis N, Mikropoulou AM, Tranos P, Dervenis P (2017) Ranibizumab in the treatment of diabetic macular edema: a review of the current status, unmet needs, and emerging challenges. Adv Ther 34:1270-1282. https://doi.org/10.1007/s12325-017-0548-1

21. Mitchell P, Bandello F, Schmidt-Erfurth U, Lang GE, Massin P, Schlingemann RO, Sutter F, Simader C, Burian G, Gerstner O, Weichselberger A, RESTORE study group (2011) The RESTORE study: ranibizumab monotherapy or combined with laser versus laser monotherapy for diabetic macular edema. Ophthalmology 118:615-625. https://doi.org/10.1016/j.ophtha.2011.01.031

22. Zucchiatti I, Bandello F (2017) Intravitreal ranibizumab in diabetic macular edema: long-term outcomes. Dev Ophthalmol 60:63-70. https://doi.org/10.1159/000460496

23. Snaith RP, Zigmond AS (1986) The hospital anxiety and depression scale. Br Med J (Clin Res Ed) 292:344. https://doi.org/10. 1136/bmj.292.6516.344

24. Kitamura T (1993) The hospital anxiety and depression scale (HADS) [In Japanese]. Archives of Psychiatric Diagnostics and Clinical Evaluation 4:371-372

25. Patrao NV, Antao S, Egan C, Omar A, Hamilton R, Hykin PG, Sivaprasad S, Rajendram R, Moorfields Diabetic Macular Edema Study Group (2016) Real-world outcomes of ranibizumab treatment for diabetic macular edema in a United Kingdom National Health Service setting. Am J Ophthalmol 172:51-57. https://doi. org/10.1016/j.ajo.2016.09.002

26. Holekamp NM, Campbell J, Almony A, Ingraham H, Marks S, Chandwani H, Cole AL, Kiss S (2018) Vision outcomes following anti-vascular endothelial growth factor treatment of diabetic macular edema in clinical practice. Am J Ophthalmol 191:83-91. https://doi.org/10.1016/j.ajo.2018.04.010

27. Ziemssen F, Wachtlin J, Kuehlewein L, Gamulescu MA, Bertelmann T, Feucht N, Voegeler J, Koch M, Liakopoulos S, SchmitzValckenberg S, Spital G, OCEAN study group (2018) Intravitreal ranibizumab therapy for diabetic macular edema in routine practice: two-year real-life data from a non-interventional, multicenter study in Germany. Diabetes Ther 9:2271-2289. https://doi.org/10. 1007/s13300-018-0513-2
28. Massin P, Creuzot-Garcher C, Kodjikian L, Girmens JF, Delcourt C, Fajnkuchen F, Glacet-Bernard A, Guillausseau PJ, Ponthieux A, Blin P, Grelaud A (2019) Real-world outcomes with ranibizumab $0.5 \mathrm{mg}$ in patients with visual impairment due to diabetic macular edema: 12-month results from the 36-month BOREALDME study. Ophthalmic Res 62:101-110. https://doi.org/10.1159/ 000497406

29. Mitchell P, Sheidow TG, Farah ME, Mahmood S, Minnella AM, Eter N, Eldem B, Al-Dhibi H, Macfadden W, Parikh S, DungerBaldauf C, Mahgoub MM, Schmidt-Erfurth U, LUMINOUS study investigators (2020) Effectiveness and safety of ranibizumab 0.5 $\mathrm{mg}$ in treatment-naive patients with diabetic macular edema: results from the real-world global LUMINOUS study. PLoS One 15:e233595. https://doi.org/10.1371/journal.pone.0233595

30. Epstein D, Amrén U (2018) Long-time outcome in patients treated with ranibizumab for diabetic macular edema: a 4-year study. Retina 38:183-186. https://doi.org/10.1097/IAE.0000000000001501

31. Shimura M, Kitano S, Muramatsu D, Fukushima H, Takamura Y, Matsumoto M, Kokado M, Kogo J, Sasaki M, Morizane Y, Kotake O, Koto T, Sonoda S, Hirano T, Ishikawa H, Mitamura Y, Okamoto F, Kinoshita T, Kimura K, Sugimoto M, Yamashiro K, Suzuki Y, Hikichi T, Washio N, Sato T, Ohkoshi K, Tsujinaka H, Kusuhara S, Kondo M, Takagi H, Murata T, Sakamoto T, Japan Clinical Retina Study (J-CREST) group (2020) Real-world management of treatment-naïve diabetic macular oedema in Japan: two-year visual outcomes with and without anti-VEGF therapy in the STREAT-DME study. Br J Ophthalmol 104:1209-1215. https://doi.org/10.1136/bjophthalmol-2019-315199

32. Blinder KJ, Dugel PU, Chen S, Jumper JM, Walt JG, Hollander DA, Scott LC (2017) Anti-VEGF treatment of diabetic macular edema in clinical practice: effectiveness and patterns of use (ECHO Study Report 1). Clin Ophthalmol 11:393-401. https:// doi.org/10.2147/OPTH.S128509

33. Sugimoto M, Tsukitome H, Okamoto F, Oshika T, Ueda T, Niki M, Mitamura Y, Ishikawa H, Gomi F, Kitano S, Noma H, Shimura M, Sonoda S, Sawada O, Ohji M, Harimoto K, Takeuchi M, Takamura Y, Kondo M, Sakamoto T (2019) Clinical preferences and trends of anti-vascular endothelial growth factor treatments for diabetic macular edema in Japan. J Diabetes Investig 10:475-483. https://doi.org/10.1111/jdi.12929

34. Kempen GIJM, Ballemans J, Ranchor AV, van Rens GHMB, Zijlstra GAR (2012) The impact of low vision on activities of daily living, symptoms of depression, feelings of anxiety and social support in community-living older adults seeking vision rehabilitation services. Qual Life Res 21:1405-1411. https://doi.org/10. 1007/s11136-011-0061-y

35. Yu Y, Feng L, Shao Y, Tu P, Wu HP, Ding X, Xiao WH (2013) Quality of life and emotional change for middle-aged and elderly patients with diabetic retinopathy. Int J Ophthalmol 6:71-74. https://doi.org/10.3980/j.issn.2222-3959.2013.01.15

Publisher's note Springer Nature remains neutral with regard to jurisdictional claims in published maps and institutional affiliations. 


\section{Authors and Affiliations}

Taiji Sakamoto $^{1}$ (1) $\cdot$ Masahiko Shimura ${ }^{2} \cdot$ Shigehiko Kitano $^{3} \cdot$ Masahito Ohji $^{4} \cdot$ Yuichiro Ogura $^{5}$. Hidetoshi Yamashita ${ }^{6} \cdot$ Makoto Suzaki $^{7} \cdot$ Kimie Mori $^{7} \cdot$ Yohei Ohashi $^{7} \cdot$ Poh Sin Yap $^{8}$. Takeumi Kaneko ${ }^{7}$. Tatsuro Ishibashi ${ }^{9} \cdot$ for the MERCURY Study Group

\section{Taiji Sakamoto}

tsakamot@m3.kufm.kagoshima-u.ac.jp

1 Department of Ophthalmology, Kagoshima University, 8-35-1 Sakuragaoka, Kagoshima 890-8544, Japan

2 Department of Ophthalmology, Tokyo Medical University Hachioji Medical Center, Tokyo, Japan

3 Diabetes Center, Tokyo Women's Medical University, Tokyo, Japan

4 Department of Ophthalmology, Shiga University of Medical Science, Otsu, Shiga, Japan
5 Department of Ophthalmology and Visual Science, Nagoya City University, Nagoya, Aichi, Japan

6 Department of Ophthalmology and Visual Sciences, Yamagata University, Yamagata, Japan

7 Medical Division, Novartis Pharma K.K., Tokyo, Japan

8 Novartis Corporation (M) Sdn. Bhd., Selangor, Malaysia

9 Department of Ophthalmology, Kyushu University, Fukuoka, Japan 